\title{
Acrylamide encephaloneuropathy due to well water pollution
}

\author{
HIDEKI IGISU, ${ }^{1}$ IKUO GOTO, YASUO KAWAMURA, MOTOHIRO KATO, \\ KANJI IZUMI, AND YOSHIGORO KUROIWA
}

From the Department of Neurology, Neurological Institute, Faculty of Medicine, Kyushu University, Fukuoka 812, Japan

\begin{abstract}
SYNOPSIS All five members of a family developed subacutely mental confusion and/or truncal ataxia. Symptoms and signs of polyneuropathy were seen later. The well water in the patients' home contained $400 \mathrm{ppm}$ acrylamide. The present cases are unique in that they are cases of acrylamide poisoning induced by oral intake and percutaneous penetration, and that central nervous system symptoms were prominent.
\end{abstract}

Acrylamide $\left(\mathrm{CH}_{2}=\mathrm{CHCONH}_{2}\right)$ is widely used in industry such as in grouting, flocculation, printing, and paper making as well as in chemical laboratories. Although the polymer is insoluble in water and believed to be non-toxic, the monomeric form is water soluble and neurotoxic (Kuperman, 1958; McCollister et al., 1964). Several reports of acrylamide poisoning in man have been published (Fujita et al., 1960; Auld and Bedwell, 1967; Garland and Patterson, 1967; Satoyoshi et al., 1971; Takahashi et al., 1971). These cases were due to occupational exposure to acrylamide in which it seemed to have invaded through the skin and/or possibly lungs, and the major symptoms were those of polyneuropathy.

Here we describe a family with severe disturbances of the central nervous system followed by the appearance of polyneuropathy which were caused by well water containing a large amount of acrylamide.

\section{CASE REPORTS}

The family lives in Shingu, a town located to the north-east of Fukuoka City. In the middle of March 1974 all the five members of the family began to show nervous symptoms, details of which are as follows:

CASE 1 A 40 year old housewife noted rhinorrhoea

Address for correspondence: Dr Hideki Igisu, at above address. (Accepted 14 January 1975.) and dizziness on 15 March. Three days later, she became unsteady on her legs. On 20 March, she began to show irrational behaviour and was unable to walk and urinate. On admission (22 March), physical examination revealed many ecchymoses, $1-2 \mathrm{~cm}$ in diameter, on the extremities. She was confused and markedly hallucinated (visual, auditory, and tactile). Orientation and memory were poor, and a tendency to confabulate was noted. The cranial nerves were intact except for mild slurred speech. Neither apparent weakness nor ataxia was found in the limbs. Deep reflexes were mildly hyperactive without any pathological reflexes. Truncal ataxia was so severe that she could not stand alone. A catheter was needed for urination, and an enema for defaecation.

The complete blood count (CBC) was normal. Erythrocyte sedimentation rate (ESR) was $19 \mathrm{~mm} / \mathrm{h}$. Serum electrolytes, total cholesterol, serum glutamic oxaloacetic transaminase (SGOT), serum glutamic pyruvic transaminase (SGPT) were normal. Blood urea nitrogen (BUN) was slightly elevated $(32 \mathrm{~g} / \mathrm{dl})$. The cerebrospinal fluid (CSF) cell count, glucose, and protein were normal. The electroencephalogram (EEG) on the fifth hospital day suggested excessive sleepiness but no abnormalitiès were found. The motor nerve conduction velocity of the ulnar nerve and the tibial nerve were normal. The sural nerve sensory conduction velocity on the sixth hospital day was $37.1 \mathrm{~m} / \mathrm{s}$ (normal: more than $38 \mathrm{~m} / \mathrm{s}$ ).

Thiamine $(500 \mathrm{mg})$ was injected daily for 20 days. The mental symptoms, dysarthria, truncal ataxia, and dysuria disappeared within one month, while mild difficulty in defaecation persisted. Two weeks 
after admission, she began to complain of numbness in the distal parts of the extremities. Decreased touch, pain, and vibration sense was found in the extremities, more prominent distally. The ankle jerks were lost. The sensory conduction velocity of the sural nerve on the 20th hospital day was apparently lowered $(35.9 \mathrm{~m} / \mathrm{s})$.

The ulnar and tibial nerve motor conduction velocities were normal. Four months later, neurological examination revealed no abnormalities except for mild dysaesthesia in the feet and slight difficulty in defaecation. The sural sensory conduction velocity had returned to normal.

CASE 2 A 42 year old man, the husband of case 1, noted coughing, rhinorrhoea, and slight unsteadiness on his legs on 14 March. A week later the gait disturbance became worse and he began to see unreal things such as 'fireballs'. On physical examination on admission (25 March), he appeared euphoric and poor in memory and understanding. Occasionally, especially when he was falling asleep, vivid visual hallucinations were noted. Cranial nerves were intact except for bilateral horizontal nystagmus and mild slurred speech. The limbs were normal in power and coordination. Deep reflexes were normally active without any pathological reflexes. No apparent sensory impairment was disclosed. He showed poor tandem gait. The sphincters were intact.

Laboratory data including CBC, urinalysis, SGOT, SGPT, BUN, and electrolytes were normal. CSF cell count and sugar were normal but the protein was mildly elevated $(70 \mathrm{mg} / \mathrm{dl})$. The motor conduction velocities of ulnar and tibial nerves and sensory conduction velocity of the sural nerve were normal. The EEG was interpreted as a normal waking and sleep record.

Hallucinations disappeared within a week after admission. One month later neither truncal ataxia nor horizontal nystagmus was noted. Twenty days after admission, he complained of dysaesthesia in the fingers. Mild decrease of touch and vibration sense was noted in the distal parts of the extremities. The ulnar and tibial motor nerve conduction velocities were normal but the sural nerve sensory conduction velocity was lowered $(35.7 \mathrm{~m} / \mathrm{s})$. Two months later the latter measurement and the neurological examination revealed no abnormalities.

CASE 3 A 65 year old woman, mother of case 2, noted coughing and sore throat on 17 March. Three days later, she developed a disturbance of gait. She became sleepy and began to see unreal things. Physical examination on admission (22 March) disclosed mild hypertension and a small number of ecchymoses in the thighs. She easily fell asleep and showed poor memory and understanding. She noted vivid visual hallucinations especially when she was drowsy. Cranial nerves were intact except for horizontal nystagmus to the left and mild dysarthria. Power and coordination of the limbs were normal but the reflexes in the right lower limb were slightly hyperactive and Babinski's sign was noted on the right. Truncal ataxia was so marked that she could not perform tandem gait. Sensation and the sphincters were normal.

Laboratory data including $\mathrm{CBC}$, urinalysis, serum electrolytes, cholesterol, SGOT, SGPT, BUN, CSF cell count, sugar, and protein were normal. The EEG suggested excessive sleepiness but no abnormalities were found. The motor and sensory nerve conduction velocities were normal. Radiological examination revealed mild spondylotic changes in the cervical spines.

Neither mental symptoms, dysarthria, nor truncal ataxia was seen on the 10th hospital day, but four weeks after admission mild sensory impairment in the limbs was noted. The ulnar and tibial nerve motor conduction velocities were normal but the sensory conduction velocity of the sural nerve was lowered $(34.9 \mathrm{~m} / \mathrm{s})$.

Two months later, no abnormalities were found on $\stackrel{\mathbb{\mathbb { D }}}{\infty}$

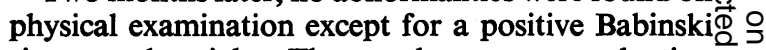
sign on the right. The sural sensory conductiongvelocity became normal.

CASE 4 A 13 year old boy, the son of case 1, began to show sleepiness and gait disturbance on 15 March. On physical examination (23 March), moderate truncal ataxia was noted. He was normal after two weeks. The motor conduction velocity of ulnar and tibial nerves and the sural sensory conduction velocity were normal.

CASE 5 A 10 year old girl, the daughter of case 1 , showed peculiar behaviour, such as asking silly questions, which lasted from 20 to 22 March. The physical examination (23 March) revealed no abnormalities.

\section{ENVIRONMENT AND ANALYSIS OF WATER}

An epidemiological study was carried out by the staffs of the Sanitation Department of Fukuoka Prefecture and by ourselves. No persons with similar symptoms were found near the patients' home.

Chemical grouting was done on 18 February 1974, for sewerage construction in the road facing the patients' home. The agent used in grouting was NITTO-SS30R which consisted of acrylamide in addition to small amounts of 1,3-diacrylamidemethyl2-imidazolidone and dimethylaminopropionitrile. 


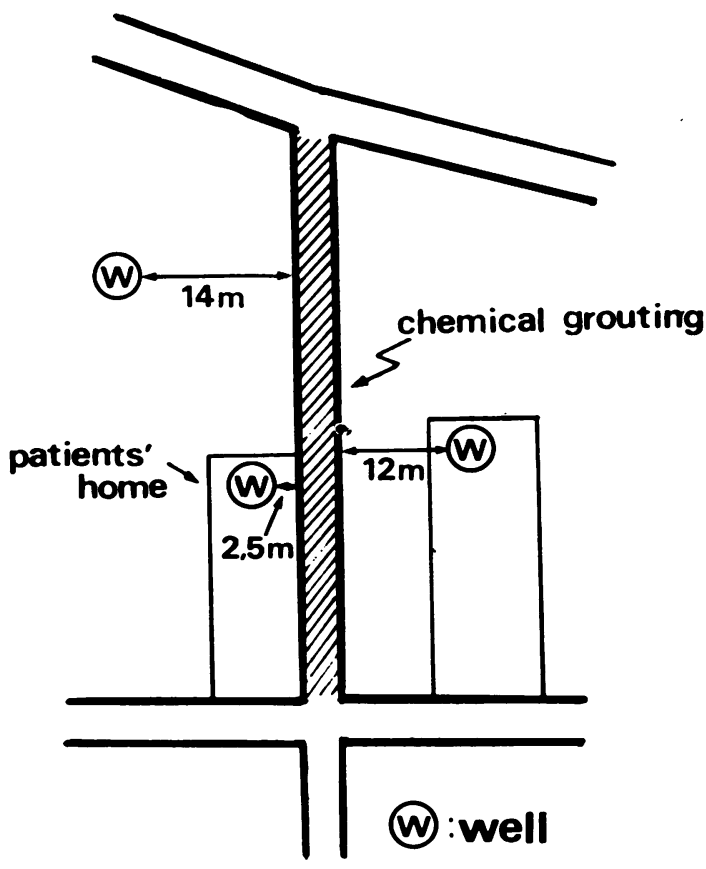

The well in the patients' home was $4 \mathrm{~m}$ in depth and $2.5 \mathrm{~m}$ apart from the road that was chemically grouted. The other two wells nearby were $12 \mathrm{~m}$ and $14 \mathrm{~m}$ apart from the road, respectively (Figure).

The well water was analysed in Fukuoka Environmental Research Centre. Gas chromatographic study of the water from the well in the patients' home, sampled on 23 March, revealed $400 \mathrm{ppm}$ acrylamide and a trace of dimethylaminopropionitrile. In the water from the other two wells, no acrylamide could be detected.

Thus, it was concluded that the acrylamide used in the grouting had infiltrated the well in the patients' home.

The patients had been using the tap water for bathing and washing and the well water for drinking and cooking because the 'taste' of the well water was better than that of the tap water. They used well water in bathing and washing also from 12 to 18 March while tap water was not supplied. Use of the well water was stopped completely on 22 March.

Case 1 and 3 stayed at home. Case 2 worked outside but he often took the well water at home with alcoholic beverages. Case 4 and 5 were at school in the daytime.

\section{ANIMAL EXPERIMENTS}

Twelve male ddN mice weighing 21-33 $\mathrm{g}$ were used. Six mice in the test group were supplied ad libitum with water from the well in the patient's home. The six in the control group were given tap water. After two months, four mice in the test group showed weakness of the limbs, more prominent in the hind limbs with the plantar surface of the paw upwards. (Two other mice in the test group were killed before paralysis appeared.)

\section{DISCUSSION}

All members of a family developed nervous symptoms subacutely within a week. The well water they used was found to contain a large amount of acrylamide, a neurotoxic agent. Our animal experiment also showed that the well water was neurotoxic. Thus, it became apparent that the encephaloneuropathy in these cases was caused by the well water polluted with acrylamide. Although the water contained dimethylaminopropionitrile, which can produce pneumonia in the rat (Bachhuber et al., 1955), the amount was so small that its effect seemed very little, if any.

It has been shown in animal experiments that neurotoxic effects of acrylamide can be produced by oral, percutaneous, intraperitoneal, or intravenous administration (McCollister et al., 1964) and that the syndrome of intoxication depends on the dose, the rate, and the length of the time of administration (Kuperman, 1958). Previously reported human cases of acrylamide poisoning (Fujita et al., 1960; Auld and Bedwell, 1967; Garland and Patterson, 1967; Satoyoshi et al., 1971; Takahashi et al., 1971), which were seen in workers handling acrylamide, were caused by its penetration through the skin and/or possibly lungs. The duration of exposure to acrylamide varied from one month (Garland and Patterson, 1967; Satoyoshi et al., 1971) to eight years (Takahashi et al., 1971). Probably much more acrylamide was absorbed by our patients via mouth and skin within one month.

The predominant symptoms and signs in the previously reported cases were those of polyneuropathy, although some showed drowsiness or ataxia associated with minor sensory impairment (Auld and Bedwell, 1967; Garland and Patterson, 1967). In our patients, the prominent symptoms were severe truncal ataxia and mental 
confusion, which suggest affection of the cerebellum and the brain-stem. The difference in symptomatology of the present cases from those previously reported is probably because these cases were of subacute intoxication induced by a large dose of acrylamide. Dysaesthesia in the limbs, absence of ankle jerks, or apparent reduction of sensory conduction velocity of the sural nerve, all of which indicate impairment of the peripheral nerve, were found after the central nervous system disturbance had almost subsided.

Thus, the present cases showed that subacute intoxication with a large dose of acrylamide can cause severe symptoms of the central nervous system and that polyneuropathy may appear later.

The symptoms in the children were less severe. This may be due to the fact that they did not take as much polluted water as the adults because they were at school in the daytime, and possibly due to the difference in susceptibility to acrylamide between children and adults which was noted in animal experiments (Fullerton and Barnes, 1966; Kaplan and Murphy, 1972).

Good recovery was seen in all cases, including the most severe, within four months. This suggests that the prognosis of acrylamide poisoning may not be poor.

DrT. Takemura referred the patients to us. We are grateful to the staffs of the Sanitation Department of Fukuoka Prefecture and Fukuoka Environmental Research Centre for their kind cooperation, and to Professor K. Tanaka (Department of Pharmacology) and Professor H. Yoshimura (Faculty of Pharmacy) for valuable comments.

\section{REFERENCES}

Auld, R. B., and Bedwell, S. F. (1967). Peripheral neuropathy with sympathetic overactivity from industrial contact with acrylamide. Canadian Medical Association Journal, 96, 652-654

Bachhuber, T. E., Lalich, J. J., Angevine, D. M., Schilling, E. D., and Strong, F. M. (1955). Lathyrus factor activity of beta-aminopropionitrile and related compounds. Proceeding of the Society for Experimental Biology and Medicine, 89, 294-297.

Fujita, A., Shibata, M., Kato, H., Ome, Y., Itomi, K., Suzuki, K., Nakazawa, T., and Takahashi, T. (1960). Clinical observations on 3 cases of acrylamide poisoning. Nippon Iji Shimpo, 37-40.

Fullerton P. M., and Barnes, J. M. (1966). Peripheral neuropathy in rats produced by acrylamide. British Journal of Industrial Medicine, 23, 210-221.

Garland, T. O., and Patterson, M. W. H. (1967). Six cases of acrylamide poisoning. British Medical Journal, 4, 134138.

Kaplan, M. L., and Murphy, S. D. (1972). Effect of acrylamide on rotarod performance and sciatic nerve $\beta$-glucuronidase activity of rats. Toxicology and Applied Pharmacology, 22, 259-268.

Kuperman, A. S. (1958). Effects of acrylamide on the centra尼 nervous system of the cat. Journal of Pharmacology and Experimental Therapeutics, 123, 180-192.

McCollister, D. D., Oyen, F., and Rowe, V. K. (1964) Toxicology of acrylamide. Toxicology and Applied Pharmacology, 6, 172-181.

Satoyoshi, E., Kinoshita, M., Yano, H., and Suzuki, Y (1971). Three cases of peripheral polyneuropathy due to acrylamide. Clinical Neurology (Tokyo), 11, 667-672.

Takahashi, M., Ohara, T., and Hashimoto, K. (1971). An' electrophysiological study on nerve injuries of workers handling acrylamide. Clinical Neurology (Tokyo), 11, 261269. 